\title{
Dinâmica de Sistemas e Prospecção de Cenários: Uma Revisão da Literatura
}

\author{
Carolina Resende Haddad \\ United Nations University-MERIT/Maastricht University, UM, The Netherlands \\ c.resendehaddad@student.maastrichtuniversity.com \\ André Catapan \\ Universidade Federal de Santa Catarina, UFSC, Brasil \\ andrecatapan@gmail.com \\ Fernando da Silva \\ Universidade Federal de Santa Catarina, UFSC, Brasil \\ fernandodasilva89@gmail.com \\ Mauricio Uriona Maldonado \\ Universidade Federal de Santa Catarina, UFSC, Brasil \\ m.uriona@ufsc.br
}

\section{RESUMO}

Pouco se discute sobre a aplicação conjunta das ferramentas de dinâmica de sistemas e de prospecção. No presente artigo, objetivase revisar a literatura no que tange à aplicação conjunta desses assuntos, por meio do uso de bibliometria e análise contextual. A partir da aplicação do método Proknow-C, foram selecionados 35 artigos das bases de dados Scopus e Scielo, abrangendo o período de 1960 a 2014, de acordo com sua relevância e relação com o tema. Os resultados apontam que os principais journals que apresentam ambos os temas são Technological Forecasting and Social Change e o Journal of the Operational Research Society. A primeira publicação analisada data de 1976 e as publicações seguintes só vieram a partir de 1988. Daim et al. e Lyneis são os autores cujos artigos tiveram o maior número de citações. As principais metodologias utilizadas foram dinâmica de sistemas e estudo de caso. Em relação à análise contextual, identifica-se que muitos artigos utilizam modelagem e simulação para prospectar o futuro e construir cenários, mas poucos discutem se essa aplicação é a mais adequada e como se devem utilizar as duas ferramentas em conjunto. 
PALAVRAS-ChaVE: Dinâmica de sistemas. Prospecção de cenários. Revisão da literatura.

\section{Systems Dynamics and Scenarios Prospecting: A Review of Literature}

\section{ABSTRACT}

Little is discussed about the joint application of systems dynamics and prospecting tools. This article aims to review the literature regarding the joint application of these subjects through the use of bibliometrics and contextual analysis. From the application of the Proknow-C method, 35 articles were selected from the Scopus and Scielo databases, covering the period from 1960 to 2014, according to their relevance and relation to the theme. The results indicate that the main journals that present both themes are "Technological Forecasting and Social Change" and "Journal of the Operational Research Society". The first publication analyzed dates from 1976 and the following publications only came from 1988. Daim et al. and Lyneis are the authors whose articles had the highest number of citations. The main methodologies used were system dynamics and case study. In relation to the contextual analysis, it is identified that many articles use modeling and simulation to prospect the future and construct scenarios, but few discuss whether this application is the most adequate and how to use the two tools together.

KEY-WORDS: Dynamics of systems. Prospecting scenarios. Literature review. 


\section{INTRODUÇÃO}

O conceito de dinâmica de sistemas tem cada vez mais sido usado por empresas, governo e instituições e aplicado às mais diversas áreas, desde o estudo do clima à psicologia (Sterman, 2007). A dinâmica de sistemas "combina teorias, métodos e filosofias para analisar o comportamento dos sistemas" (Geum, Lee \& Park, 2014, p. 39).

A prospecção de cenários permite expandir as possibilidades do que está sendo pesquisado, o que é feito dividindo o conhecimento entre o que os pesquisadores acreditam saber e o que é desconhecido (Schoemaker, 1995). Além disso, os cenários procuram antecipar mudanças no ambiente e aceitar incertezas, o que deve ser considerado no processo de previsão (Wack, 1985, citado por Huss \& Honton, 1987).

A dinâmica de sistemas foca no comportamento de um sistema no presente, enquanto a prospecção de cenário foca no que pode ocorrer no futuro, a partir de um entendimento do presente (Featherson \& Doolan, 2013). Esses autores propõem o uso da metodologia de cenários para mapear o sistema e da dinâmica de sistemas para especificar as causas do comportamento desse sistema. Randers e Göluke (2007), por outro lado, questionam se a dinâmica de sistemas pode e deve ser usada para prospectar futuros. A opinião dos autores é que elas podem e devem ser usadas em conjunto, desde que o sistema seja bem estruturado.

Featherston e Doolan (2013) e Randers e Göluke (2007) identificaram algumas das lacunas na literatura sobre a aplicação conjunta das metodologias de prospecção e dinâmica de sistemas. Isso evidencia a falta de estudos que explicam e discutem o desenvolvimento da aplicação conjunta desses temas ao longo dos anos. Dessa forma, no presente artigo, objetiva-se revisar a literatura sobre aplicação conjunta de dinâmica de sistemas e prospecção. No artigo, apresentam-se os resultados dessa revisão, com base na análise bibliométrica e contextual dos artigos selecionados.

Foi realizada a análise sistemática de 35 artigos, selecionados a partir de um montante de 212 artigos, por meio da aplicação do método Proknow-C. Primeiramente, foram selecionados os artigos publicados entre 
1960 e 2014 na Scopus, por esta ser a maior base de dados de resumos e citações na área de ciências, tecnologia e ciências sociais (Elsevier, 2014). Paralelamente, fez-se uso da base de dados Scielo para pesquisa e obtenção de artigos nacionais.

Os resultados incluem a análise cronológica dos artigos selecionados, bem como a relação dos autores, artigos e instituições mais relevantes sobre o assunto. Além disso, na sequência, há uma análise semântica e contextual dos artigos, que identifica as palavras-chave e metodologias mais usadas e as principais propostas de estudos futuros sobre dinâmica de sistemas, prospecção e dinâmica de sistemas em conjunto com prospecção.

O artigo é estruturado em cinco seções. Na seção 1, apresenta-se uma introdução sobre o contexto e o objetivo da pesquisa. Na seção 2, fazse uma contextualização sobre prospecção e dinâmica de sistemas. Na próxima seguinte, relacionam-se os aspectos metodológicos adotados. Em seguida, na seção 4, trazem-se os principais resultados encontrados. Por fim, na seção 5, apresentam-se os principais resultados e contribuições.

\section{REVISÃO TEÓRICA}

\subsection{DINÂMICA DE SISTEMAS}

De acordo com Bueno (2013), a dinâmica de sistemas é uma metodologia desenvolvida para analisar as relações de causa e efeito que determinadas variáveis possuem num sistema, seja ele organizacional ou social. Essa metodologia é bastante ligada ao pensamento sistêmico e busca representar essas relações por meio do estudo de sua evolução ao longo do tempo (Figueiredo, 2009). A partir de padrões de comportamento, busca-se compreender e explicar o comportamento de variáveis isoladas ou em conjunto num sistema existente (Sterman, 2000; Pidd, 1998; Forrester, 1961).

Para Figueiredo (2009), a dinâmica de sistemas assume que a análise de uma situação pode ser empreendida de um ponto de vista externo ao sistema, e que a estrutura e os processos dinâmicos podem ser 
recriados em diagramas com simbologia específica e modelos matemáticos apropriados. Essa análise é feita em softwares de simulação, que permitem o teste de diferentes políticas e soluções para a operação de um sistema, avaliando o impacto de suas decisões e proporcionando um conjunto de instrumentos para compreensão e comunicação sobre os modelos construídos (Pidd, 1998).

Figueiredo (2009) afirma ainda que um comportamento dinâmico surge quando algo flui por algum meio, ou seja, a resposta dinâmica de um sistema decorre da transição dos valores de seus recursos acumulados em estoques, e que essas transições são controladas por fluxos de entrada e saída nos estoques. Bueno (2013), por sua vez, afirma que novas informações levam a ações (fluxos) que alteram o estado (nível) das condições de um sistema após uma defasagem de tempo. Ação, nível e informação interligam-se em dois tipos de ciclos de feedback. O primeiro tipo é o ciclo de feedback negativo ou de equilíbrio em que o sistema reage a mudanças compensando-as. O segundo tipo é o ciclo de feedback positivo ou de autorreforço, em que o sistema amplifica eventuais perturbações.

As principais vantagens advindas do uso da metodologia de dinâmica de sistemas provêm de sua capacidade de: a) investigar as relações entre macro e microestruturas e seus efeitos sobre o comportamento do sistema; b) modelar e resolver problemas reais, incorporando fatores biológicos, físicos e econômicos; c) melhorar o desempenho de um sistema via adição de insights ou aprendizagem, aliado ao melhor uso dos recursos; d) estudar os fluxos de material, informação e dinheiro dentro de estruturas econômicas; e) não ter um limite dos problemas que possa abranger, podendo captar situações de equilíbrio, desequilíbrio e até mesmo comportamentos caóticos. Frente a essas habilidades, a metodologia de dinâmica de sistemas vem sendo aplicada nas mais diversas áreas do conhecimento (Figueiredo, 2009).

\subsection{PROSPECÇÃO E ANÁLISE DE CENÁRIOS}

Bradfield, Wright, Burt, Cairns e Van Der Heijden (2005) trazem uma revisão das origens e evolução do uso de técnicas de cenários, 
apontando que a literatura possui diferentes definições e metodologias para o tema. De acordo com os autores, as técnicas para construção de cenários emergiram no pós-guerra, com o desenvolvimento de dois centros geográficos principais na década de 1960: Estados Unidos e França. Nos Estados Unidos, os principais centros de estudo sobre cenários que surgiram foram a Intuitive logics school e a Probabilistic modified trends school. Na França, surgiu a Prospective school.

Herman Kahn originalmente foi o responsável pela introdução do conceito de cenário (Bishop, Hines \& Collins, 2007) em estudos futuros nos Estados Unidos, e Datar na França (Godet, 2000). Herman (1960, citado por Schnaars, 1987) escreve que o termo cenário foi usado inicialmente pelo grupo Rand Corporation, no sentido militar e estratégico, em conexão com os estudos desenvolvidos pela organização para o governo na década de 1950 (Zentner, 1982).

Segundo Bradfield et al. (2005), Helmer e outros envolvidos no Stanford Research Institute "Future Groups" (SRI) e no California Institute of Technology começaram a usar cenários como uma ferramenta de planejamento de políticas públicas. Logo em seguida, a técnica começou a ser usada no ambiente corporativo, com a Shell. A empresa foi responsável pelo desenvolvimento de um projeto para estudar o ambiente de negócios, conhecido como Intuitive logics school. A Probabilistic modified trends school, por sua vez, divide-se em duas metodologias principais: Trendimpact analysis (TIA), que faz a análise de dados históricos e o estudo de tendências, e a Cross-imapact analysis (CIA), que avalia as alterações nas ocorrências de eventos que podem causar desvios. Já a francesa Prospective school começou a desenvolver o estudo de probabilidades baseadas em matemática e computação para o desenvolvimento de cenários.

Apesar da dificuldade de encontrar uma definição, o entendimento geral para análise de cenários relaciona-se à capacidade das organizações em lidar com incertezas (Geum et al., 2014). O estudo de cenários é uma ferramenta de previsão de longo prazo em ambientes de grandes incertezas, que envolve ainda a análise de tendências e a simulação de modelos para a elaboração de planos estratégicos (Huss \& Honton, 1987). 
O planejamento de cenários é uma ferramenta que procura sintetizar saídas e identificar padrões diante de milhões de possibilidade que uma simulação pode gerar (Schomaker, 1995). O seu desenvolvimento possibilita ao pesquisador focar nas atividades-chave, nas possibilidades de evolução e nos aspectos que devem ser confrontados (Saritas \& Aylen, 2010).

Loveridge (2008, citado por Saritas \& Aylen, 2010) propõe nove etapas para o desenvolvimento de cenários:

- definir o objetivo do cenário e seus aspectos temporais;

- definir os indicadores, tais como os aspectos sociais, tecnológicos, econômicos, ecológicos, políticos e de valores;

- analisar os assuntos que serão usados nos cenários;

- definir alternativas e tendências;

- escrever os cenários

- analisar os cenários

- a partir da análise, identificar os aspectos que serão trabalhados;

- desenvolver estratégias

- a partir da modelagem e da simulação, avaliar as estratégias considerando tempo e alocação dos recursos.

Bishop et al. (2007), com base em uma revisão de literatura, listaram oito tipos genéricos de cenários, com duas a três variações para cada tipo (Figura 1). Conforme descrito por Börjeson, Höjer, Dreborg, Ekvall, Finnveden (2006), não há consenso sobre as tipologias de cenário, mas algumas delas refletem o que pode ser explorado sobre o assunto em estudos futuros. 


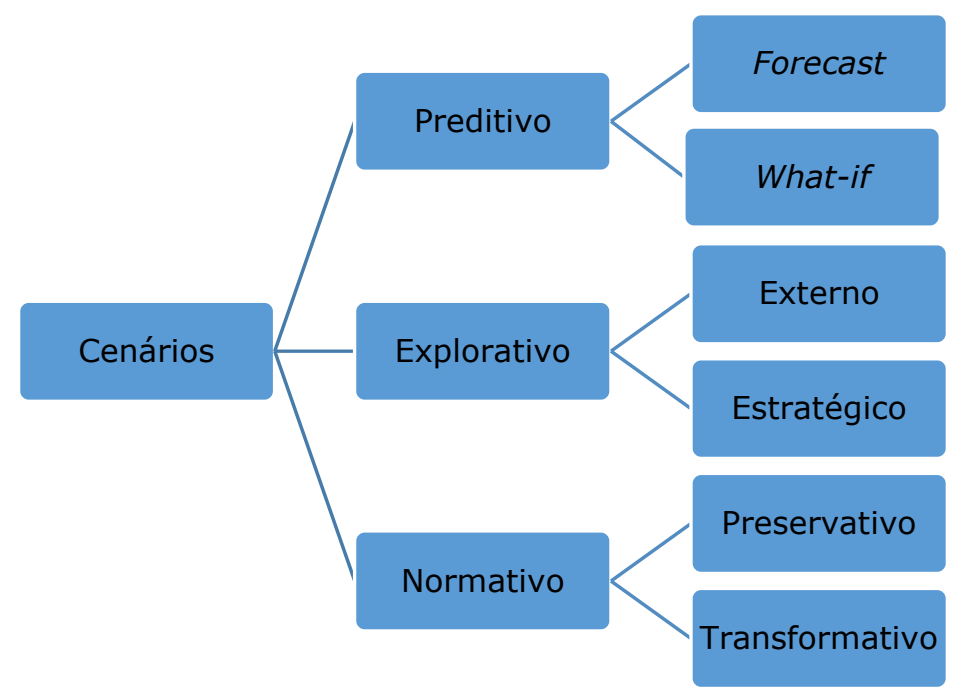

\section{Figura 1: Tipologia de cenários}

Fonte: Börjeson et al. (2006, p. 725)

Com o desenvolvimento da abordagem de cenários, outros termos começaram a ser usados no sentido de prospecção: foresight e forecasting (Cuhls, 2003). Foresight tem o objetivo de fazer um levantamento sistemático sobre como o assunto da pesquisa pode evoluir, considerando seu estado atual e quais os possíveis resultados de diferentes futuros (Solem, 1986, citado por Martin, 2010). De acordo com o Ministério de Pesquisa, Ciência e Tecnologia (1997, citado por Martin \& Johnston, 1999), um foresight objetiva, considerando diferentes indicadores, incertezas e influências, construir uma previsão sobre como o futuro irá se desenvolver.

O termo forecast, por sua vez, é usado para demonstrar o que pode acontecer caso a previsão se confirme, sendo o cenário resultante o mais provável de ser desencadeado (Börjeson et al., 2006). Segundo Wills (1972, citado por Martin, 2010), forecasting corresponde a uma afirmação probabilística de alto nível sobre o futuro. Para Martino (1993), um forecast de tecnologia possui quatro elementos essenciais: a tecnologia foco da previsão, o tempo considerado, as características dessa tecnologia e uma afirmação referente à probabilidade de ocorrência de um fato.

\section{METODOLOGIA}


A construção do portfólio bibliográfico fez uso do método ProknowC, apresentado por Ensslin et al. (2010). O Proknow-C é amplamente utilizado para a realização de estudos bibliométricos e sistêmicos e apresenta aplicações nas mais diversas áreas do conhecimento (Costa \& Marinho, 2017). A partir dos artigos selecionados, fez-se então uma análise bibliométrica, buscando identificar padrões e tendências nas publicações. Por fim, utilizou-se uma análise semântica e de conteúdo, a fim de analisar quais os tipos de pesquisas e áreas do conhecimento mais abordadas.

A construção do portfólio bibliográfico consiste de oito etapas: definição das bases de dados, definição de palavras-chave, busca e filtragem na base de dados, seleção de artigos por alinhamento de título à pesquisa, seleção por reconhecimento científico, repescagem de referências excluídas, leitura de resumos e, por fim, seleção dos artigos a compor o portfólio.

Escolheu-se, primeiramente, a base de dados Scopus para a realização da pesquisa, haja vista que consiste na maior base de citações e resumos de literatura científica, apresentando uma visão compreensiva das pesquisas no campo da ciência, tecnologia, medicina, ciências sociais e humanidades (Elsevier, 2017). Utilizou-se, paralelamente, a base de dados Scielo para obtenção dos artigos nacionais. Foram definidos dois grupos de palavras-chave: o primeiro aborda o conceito de dinâmica de sistemas ("system dynamics") e o segundo trata da previsão e prospecção de cenários ("forecast*" OR "foresight*" OR "scenario planning"). Os procedimentos específicos encontram-se na Tabela 1. 


\section{Tabela 1: Procedimentos metodológicos}

\begin{tabular}{|c|c|}
\hline Etapas & Scopus \\
\hline Busca e Filtragem & $\begin{array}{l}\text { Filtros: } \\
\text {-Subárea de pesquisa: Business, Management and } \\
\text { Accounting; Engineering; Decision Sciences; Social Sciences; } \\
\text { Economics, Econometrics and Finance; Multidisciplinary; } \\
\text { Undefined; } \\
\text {-Idioma: inglês e português; } \\
\text {-Tipo de documento: periódico; } \\
\text {-Ano de publicação: início em 1971; } \\
\text { Resultado: } 214 \text { artigos. }\end{array}$ \\
\hline $\begin{array}{ll}\text { Seleção de artigos } & \text { por } \\
\text { alinhamento do título } & \end{array}$ & Resultado: 100 \\
\hline $\begin{array}{l}\text { Seleção por reconhecimento } \\
\text { científico }\end{array}$ & $\begin{array}{l}\text { Busca por citações: Google Acadêmico } \\
\text { Regra de Pareto (1987-2009): 80,00\% das citações } \\
\text { corresponderam a } 28 \% \text { das referências; } \\
\text { Resultado: } 26 \text { artigos }\end{array}$ \\
\hline $\begin{array}{l}\text { Repescagem de referências } \\
\text { excluídas }\end{array}$ & $\begin{array}{l}\text { Seleção por título de interesse: Aqueles que tratam de } \\
\text { conceitos e características e aplicações. } \\
\text { Resultado: } 9\end{array}$ \\
\hline Leitura de resumos & $\begin{array}{l}\text { Critérios: conceitos, características, vantagens/desvantagens } \\
\text { ou dificuldades/facilidades; estudos de caso. } \\
\text { Total de resumos lidos: } 35\end{array}$ \\
\hline $\begin{array}{l}\text { Seleção dos artigos para } \\
\text { compor o portfólio de leitura }\end{array}$ & Resultado: 35 \\
\hline
\end{tabular}

Fonte: elaborado pelos autores

A análise dos dados se deu, sobretudo, a partir do uso de procedimentos bibliométricos e análise contextual. Para analisar informações decorrentes da literatura, a bibliometria utiliza métodos quantitativos, tais como técnicas estatísticas e matemáticas, a fim de gerar uma avaliação objetiva da produção científica (Araújo, 2006). De acordo com White e McCain (1989, citados por Hood \& Wilson, 2001), a bibliometria tem a finalidade de fornecer modelos evolutivos relacionados à ciência e à tecnologia.

Para Daim, Rueda, Martin e Gerdsri (2006), a bibliometria ajuda a explorar, organizar e analisar dados históricos referentes à produção acadêmica, a fim de identificar padrões na literatura. Os autores também indicam que as principais ferramentas usadas na bibliometria são, por exemplo, análise de citações, autores e instituições. No presente artigo, foram analisados: distribuição cronológica; relevância do autor em relação ao número de citações e número de artigos publicados; número de 
publicações por journal e por país; e instituições que mais publicaram e mais foram citadas.

Para a análise contextual, foram consideradas as palavras-chave, as metodologias apresentadas e as oportunidades de pesquisa apontadas pelos autores analisados. Esta análise classifica o texto publicado, refinando-o e transformando as informações em dados relevantes e maleáveis (Weber, 1990). Além disso, sua finalidade é analisar os dados sob um contexto específico, a fim de identificar os significados atribuídos a eles (Krippendorff, 2013). Ainda de acordo com o autor, a análise contextual possui três características principais: é um método empírico, visa simplificar o conteúdo e as intenções e, por fim, permite que o pesquisador planeje, execute, comunique e reproduza os resultados.

Para a análise das palavras-chave foram primeiramente consideradas aquelas sinônimas à dinâmica de sistemas e prospecção. Em seguida, as demais palavras-chave foram classificadas em 11 assuntos ou áreas: meio ambiente; logística; indústria; mercado/econômico; matemática; tecnologia; recursos humanos; riscos; gerenciamento; inovação; governança. Uma palavra-chave pode fazer parte de mais de um assunto ou área.

Em seguida, foram analisadas as metodologias utilizadas nos artigos, os quais podem possuir uma ou mais metodologias. Por fim, foram analisadas as oportunidades de pesquisa identificadas nos artigos, no que tange à dinâmica de sistemas, à prospecção e ao uso conjunto das mesmas.

\section{RESULTADOS}

\subsection{BIBLIOMETRIA}

Nesta seção, apresentam-se os principais resultados da análise bibliométrica realizada em torno da área de conhecimento de simulação e prospecção de cenários. Tal análise contempla inicialmente um breve sumário sobre os dados quantitativos da pesquisa, seguido de uma distribuição cronológica dos artigos de grande impacto, publicados ao longo das últimas cinco décadas. Posteriormente, o estudo abrange os autores e 
os artigos mais relevantes no assunto, os journals científicos mais importantes, as instituições e países mais relevantes em simulação e prospecção de cenários.

A pesquisa deu-se inicialmente com o mapeamento de todos os artigos na área de conhecimento de simulação e prospecção na base de dados Scopus. Posteriormente, considerando-se desde a primeira publicação no assunto até os dias atuais, foram filtrados desse montante os artigos mais relevantes na área, ou seja, escolheram-se para análise as obras com maiores números de citações. Nesse contexto, no estudo consideraram-se artigos de grande impacto os que obtiveram número significativo de citações após sua publicação.

Tabela 2: Sumário dos resultados gerais

\begin{tabular}{lc}
\hline Critério & Quantidade \\
\hline Artigos & 35 \\
\hline Autores & 91 \\
\hline Journals & 24 \\
\hline Países & 25 \\
\hline Instituições & 59 \\
\hline Citações & 1513 \\
\hline
\end{tabular}

Fonte: elaborado pelos autores

De acordo com a Tabela 2, das 212 obras inicialmente mapeadas, 35 artigos com o total de 1.513 citações foram escolhidos para análise, as quais contemplaram 91 autores, 24 journals e 49 instituições distribuídas em 25 países. A maioria dos artigos analisados foi escrita por mais de um autor, muitas vezes de países e instituições diferentes. Assim, para os dados analisados na Tabela 2, todos os autores, países e instituições foram levados em consideração.

\subsubsection{Distribuição cronológica}

Uma vez definido o tema de pesquisa e selecionados os artigos para estudo, outra análise importante diz respeito à distribuição cronológica das publicações na área de simulação e prospecção de cenários. Tal análise indica como a área de conhecimento vem evoluindo ao longo das últimas 
décadas com relação aos seus conceitos e aplicações e qual o grau de importância que o tema vem adquirindo entre especialistas e instituições acadêmicas.

No Gráfico 1, apresentam-se os resultados desta análise, que evidenciam que nos últimos anos houve uma notável evolução nas publicações de artigos de grande impacto ao redor do mundo. Gordon e Stoven (1976) foram os primeiros autores a terem um artigo de grande impacto, publicado no Technological Forecasting and Social Change. O artigo, intitulado "Usando as percepções e dados sobre o futuro para melhorar a simulação de sistemas complexos", abordava duas técnicas de previsão de futuro conhecidas como análise de impacto de tendência e sistema de probabilidade dinâmica. Seu objetivo era apresentar essas técnicas, visto que, na época, as pessoas tomavam decisões sobre o futuro em determinado assunto baseando-se apenas na hipótese de que o comportamento futuro seria uma extensão do passado, sem considerar fatores aleatórios.

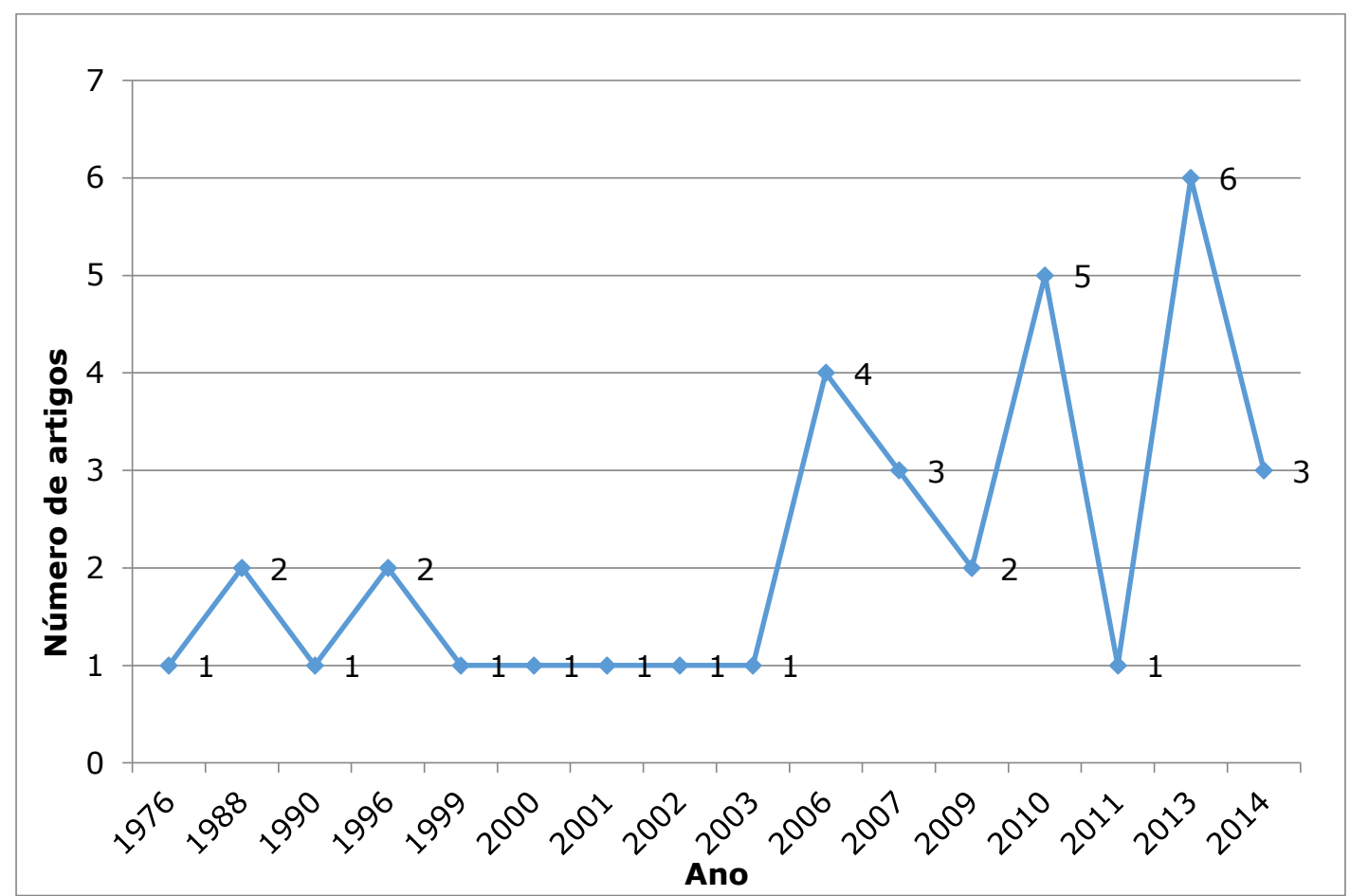

Gráfico 1: Distribuição cronológica de pesquisa de grande impacto
em simulação de prospecção de cenários

Fonte: elaborado pelos autores 
Mesmo antes dessa primeira publicação de grande impacto, porém, o tema simulação e prospecção de cenários já era alvo de estudos ao redor no mundo. Blackman, no ano de 1971, publicou o primeiro artigo na área, intitulado "Forecasting through dynamic modeling". Quatro anos mais tarde, mais três obras foram registradas. No entanto, como no presente estudo abrangem-se apenas os artigos de grande impacto oriundos do respectivo número de citações, tais obras não foram mapeadas.

Após a primeira publicação de grande impacto em 1976, nas três décadas seguintes, apresentou-se certa estabilidade na quantidade de publicações, dentre as quais é possível destacar Sterman (1988), com sua obra "Modeling the formation of expectations: The history of energy demand forecasts"; Lyneis (2000), com o artigo "System dynamics for market forecasting and structural analysis"; e Karavezyris, Timpe e Marzi (2002), com sua obra "Application of system dynamics and fuzzy logic to forecasting of municipal solid waste".

Já em 2006, houve o primeiro aumento significativo de publicações de grande impacto na área de simulação e prospecção de cenários, com quatro grandes obras. Dentre elas, merece destaque aquela escrita por Daim et al. (2006), citada 328 vezes, servindo de base para várias outras obras. O artigo de Daim et al., intitulado "Forecasting emerging technologies: Use of bibliometrics and patent analysis", aborda técnicas de previsão baseadas em bibliometria e análise de patentes para prever tecnologias emergentes.

Após o ano de 2006, as publicações na área apresentaram certa instabilidade. No entanto, mantiveram uma taxa de publicação em torno de três a quatro publicações de grande impacto por ano, até que, em 2013, foi obtido o maior número de publicações na área, contemplando seis obras de grande impacto. Embora publicadas em várias regiões do mundo, todas elas apresentaram análises ou técnicas de previsão do futuro. Podem-se destacar nesse ano os artigos de Kwakkel e Pruyt (2013), "Exploratory modeling and analysis, an approach for model-based foresight under deep uncertainty"; e de Lakka, Michalakelis, Varoutas e Martakos (2013), "Competitive dynamics in the operating systems market: Modeling and policy implications". 


\subsubsection{Relevância do autor}

Nesta análise, procurou-se mensurar a quantidade de artigos que cada autor possui, bem como a quantidade de citações que tem na área de simulação e prospecção de cenários. Diante do exposto e do fato de que existem artigos publicados na área desde a década de 1970, pode-se observar na Tabela 3 que não há uma quantidade significativa de autores, em comparação com outras áreas de conhecimento da engenharia e afins.

Tabela 3: Quantidade de publicações por autor

\begin{tabular}{ccc}
\hline $\begin{array}{c}\text { Quantidade de } \\
\text { Autores }\end{array}$ & $\begin{array}{c}\text { \% dos } \\
\text { Autores }\end{array}$ & $\begin{array}{c}\text { Quantidade de } \\
\text { Artigos }\end{array}$ \\
\hline 88 & $97 \%$ & 1 \\
\hline 3 & $3 \%$ & 2 \\
\hline
\end{tabular}

Fonte: elaborado pelos autores

Na Tabela 3, nota-se que, dos 91 autores com publicações na área de estudo, 97\% tem apenas um artigo de grande impacto. Nos 3\% dos autores restantes, percebe-se que também não há uma quantidade significativa de artigos publicados na área, apenas dois.

Dentre os $97 \%$ dos autores com apenas uma publicação de grande impacto na área de estudo do presente artigo, além da obra de Daim et al. (2006), com um número significativo de citações apresentado no tópico distribuição cronológica, pode-se destacar o trabalho realizado por Lyneis (2000). Tal obra, intitulada de "System dynamics for market forecasting and structural analysis", apresenta modelos de dinâmica de sistemas que fornecem um meio de entender as causas do comportamento da indústria e, assim, permitir a detecção precoce de alterações na sua estrutura. Esse artigo foi de grande importância para a área de estudo, pois vem sendo, até os dias atuais, referência para outras publicações em simulação e prospecção de cenários. Até o presente estudo, essa obra já possui mais de 152 citações em trabalhos publicados ao redor do mundo.

No que se refere aos três autores com mais de uma publicação sobre o assunto, totalizando $3 \%$ do total dos autores, cabe destacar todos: Suryani, Chou e Chen (2010), três autores naturais Taiwan, trabalharam juntos em duas obras. A primeira, intitulada "Air passenger demand 
forecasting and passenger terminal capacity expansion: A system dynamics framework", trata de como desenvolver um modelo para prever a demanda de passageiros aéreos e avaliar alguns cenários políticos relacionados com a expansão da capacidade do terminal de passageiros e pista para atender à demanda futura.

Já a segunda obra dos autores, juntamente com o autor da Indonésia Hartono, também publicada no ano de 2010, é o artigo "Demand scenario analysis and planned capacity expansion: A system dynamics framework", o qual aborda estratégias para desenvolver modelos de previsão de demanda e avaliação de cenários de políticas relacionadas com a expansão da capacidade planejada para atender a projeções de demanda futura otimistas e pessimistas. Os dois trabalhos, juntos, obtiveram mais de 60 citações ao redor no mundo. 
Tabela 4: Ranking dos autores com mais citações

\begin{tabular}{|c|c|c|c|c|}
\hline Ranking & Autor & Artigos & Ano & Citações \\
\hline 1 & $\begin{array}{l}\text { Daim, Rueda, } \\
\text { Martin e Gerdsri }\end{array}$ & $\begin{array}{l}\text { Forecasting emerging technologies: Use of } \\
\text { bibliometrics and patent analysis }\end{array}$ & 2006 & 328 \\
\hline 2 & Lyneis & $\begin{array}{c}\text { System dynamics for market forecasting and } \\
\text { structural analysis }\end{array}$ & 2000 & 153 \\
\hline 3 & $\begin{array}{l}\text { Karavezyris, } \\
\text { Timpe e Marzi }\end{array}$ & $\begin{array}{l}\text { Application of system dynamics and fuzzy } \\
\text { logic to forecasting of municipal solid waste }\end{array}$ & 2002 & 80 \\
\hline 4 & $\begin{array}{l}\text { Liehr, Größler, } \\
\text { Klein e Milling, }\end{array}$ & $\begin{array}{c}\text { Cycles in the sky: Understanding and } \\
\text { managing business cycles in the airline } \\
\text { market }\end{array}$ & 2001 & 71 \\
\hline 5 & Warr e Ayres & $\begin{array}{l}\text { REXS: A forecasting model for assessing the } \\
\text { impact of natural resource consumption and } \\
\text { technological change on economic growth }\end{array}$ & 2006 & 54 \\
\hline 6 & $\begin{array}{l}\text { Rousseaux, Van } \\
\text { Cutsem e Dy } \\
\text { Liacco }\end{array}$ & Whither dynamic state estimation? & 1990 & 51 \\
\hline 7 & Kumar e Yamaoka & $\begin{array}{l}\text { System dynamics study of the Japanese } \\
\text { automotive industry closed loop supply chain }\end{array}$ & 2007 & 49 \\
\hline 8 & $\begin{array}{l}\text { Syntetos, Boylan } \\
\text { e Disney }\end{array}$ & $\begin{array}{l}\text { Forecasting for inventory planning: A 50- } \\
\text { year review }\end{array}$ & 2009 & 49 \\
\hline 9 & $\begin{array}{l}\text { Wu, Kefan, Hua, } \\
\text { Shi e Olson }\end{array}$ & $\begin{array}{c}\text { Modeling technological innovation risks of an } \\
\text { entrepreneurial team using system } \\
\text { dynamics: An agent-based perspective }\end{array}$ & 2010 & 49 \\
\hline 10 & Kamath e Roy & $\begin{array}{c}\text { Capacity augmentation of a supply chain for } \\
\text { a short lifecycle product: A system dynamics } \\
\text { framework }\end{array}$ & 2007 & 45 \\
\hline
\end{tabular}

Fonte: elaborado pelos autores

Dos 35 artigos analisados na pesquisa, na Tabela 4, apresentam-se os dez primeiros colocados, no que diz respeito ao número de citações vinculadas a cada autor. É possível destacar, além dos autores Daim et al. (2006) e Lyneis (2000) já mencionados, o trabalho realizado por Karavezyris et al. (2002), que recebeu 80 citações. O artigo "Application of system dynamics and fuzzy logic to forecasting of municipal solid waste" apresenta um modelo de sistemas de gestão de resíduos baseado em dinâmica do sistema. Os demais autores citados na Tabela 4 não obtiveram diferenciação uns dos outros, uma vez que a taxa de citação girou em torno de 50 citações por artigo/autor.

\subsubsection{Journals}


Os 35 artigos selecionados estão distribuídos em 25 periódicos distintos, o que evidencia que não há uma concentração expressiva nos temas pesquisados, o que muito provavelmente se deve ao fato de que são conteúdos mais abrangentes e que podem ser utilizados nas mais diversas áreas do conhecimento. Muito embora os resultados sejam dispersos, é possível inferir os periódicos mais relevantes na área de dinâmica de sistemas e prospecção: três dos 25 periódicos analisados publicaram mais de um terço dos artigos mais citados na área.

\section{Journals X Quantidade de Artigos}

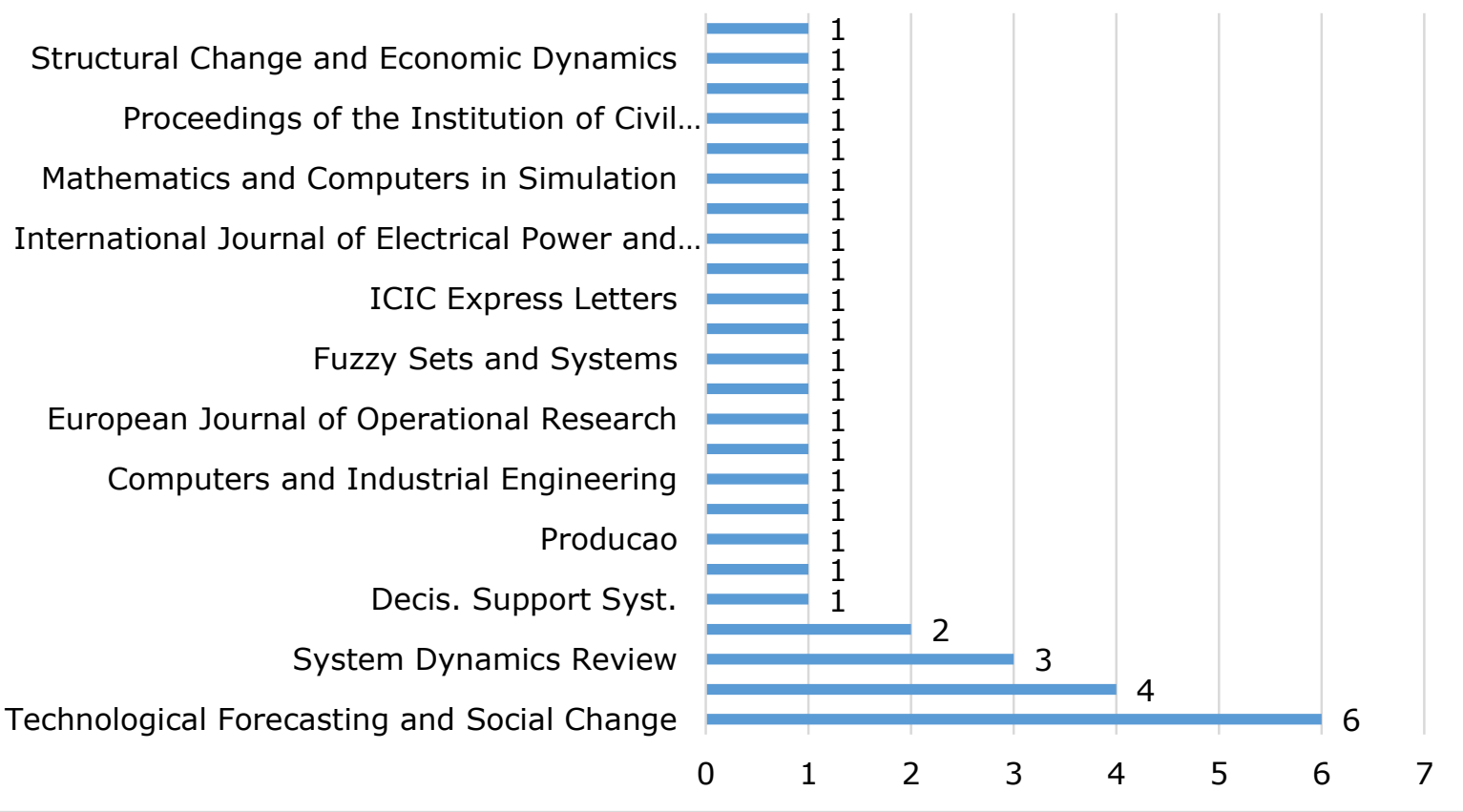

\section{Gráfico 2: Quantidade de artigos publicados por journal}

Fonte: elaborado pelos autores

Observa-se no Gráfico 2 que os três periódicos mais relevantes são aqueles que mais se assemelham com o objeto de pesquisa (Decision Support System, System Dynamics Review e Technological Forecasting and Social Change). Além disso, neles é que são publicados os avanços mais relevantes no processo de prospecção de cenários a partir da dinâmica de sistemas, enquanto nos outros são mais aceitos estudos que tratem de aplicações práticas para áreas específicas. O fator de impacto dessas publicações, principalmente da revista Technological Forecasting and Social Change $(1,959)$, acrescido da quantidade de artigos encontrados, evidencia 
a importância que esses periódicos possuem junto ao campo de estudo e demonstra que os artigos encontrados não se agruparam ao acaso.

\subsubsection{Países}

Ao se analisarem os artigos selecionados sobre a ótica dos autores e, consequentemente, países responsáveis pelas publicações, percebe-se, no Gráfico 3, uma grande gama de países envolvidos. Nesta análise, considera-se a quantidade de autores e não de artigos, pois várias obras foram desenvolvidas por um conjunto de autores de diferentes nacionalidades. Por exemplo, o artigo "Whither dynamic state estimation?" foi elaborado pelo americano Dy Liacco e pelos belgas Rousseaux e Van Cutsem (1990). Neste caso, Bélgica e Estados Unidos foram os responsáveis pelo artigo. No Gráfico 3, nota-se que os países que mais publicaram artigos de grande impacto na área de simulação e prospecção de cenários foram os Estados Unidos, Reino Unido e Alemanha.

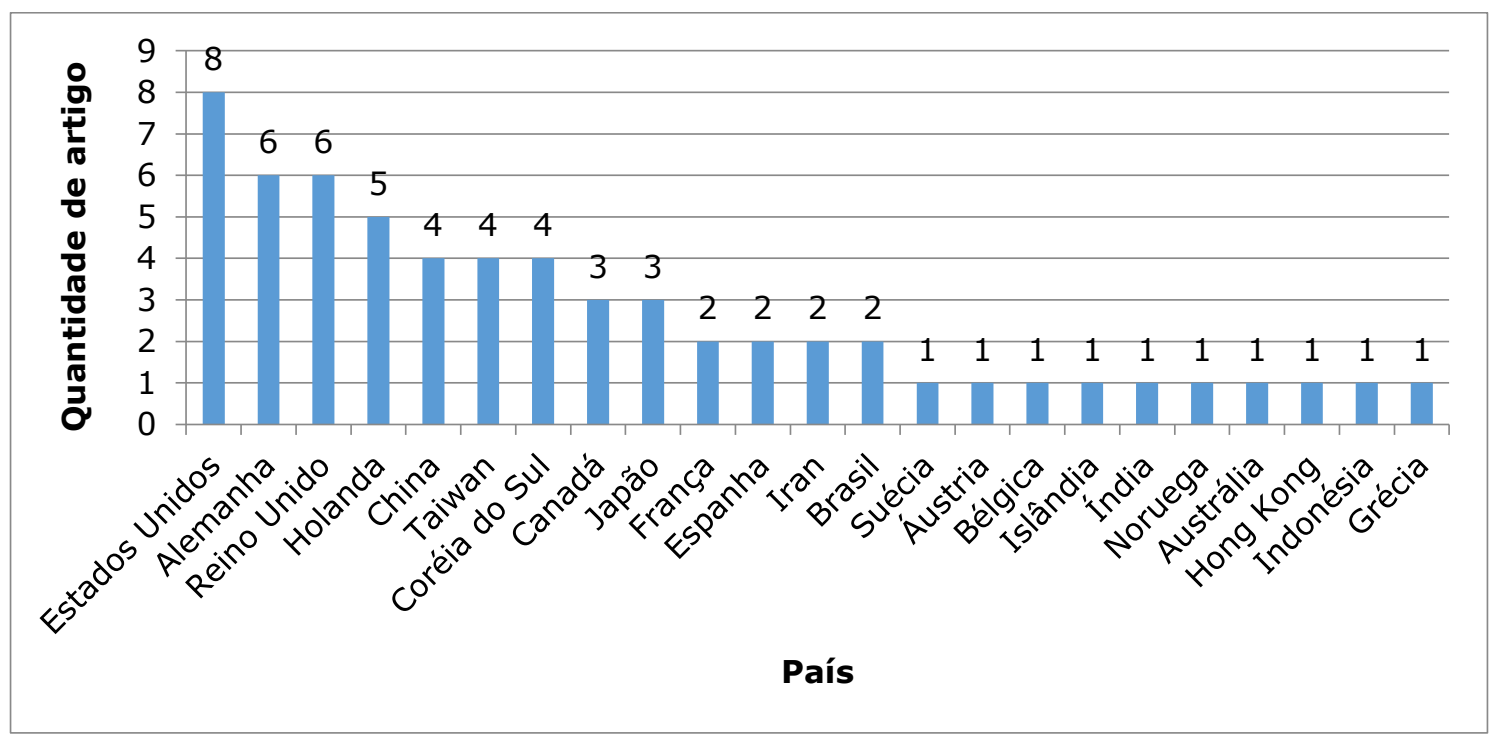

\section{Gráfico 3: Quantidade de publicações de artigos por país}

Fonte: elaborado pelos autores

\subsubsection{Instituições}

Na Tabela 5, apresentam-se as 20 instituições mais citadas, ou seja, com 45 ou mais citações. Observa-se que as instituições mais relevantes são europeias e norte-americanas, que apresentam um total de 10 e 6 
artigos mais citados, respectivamente. Em relação à produtividade, percebe-se que as instituições europeias e norte-americanas ainda se destacam. Observa-se que as únicas instituições que tiveram mais de um artigo na área são as chinesas National Taiwan University of Science and Technology e Fo Guang University.

A Portland State University apresenta-se como a instituição mais citada, com 328 citações. Em seguida, encontra-se outra universidade estadunidense, a Cambridge University, com o total de 153 citações. As duas universidades na sequência com maior número de citações são alemãs, a Technical University of Berlin e a Mannheim University, com 80 e 71 citações, respectivamente. 
Tabela 5: Instituições mais citadas

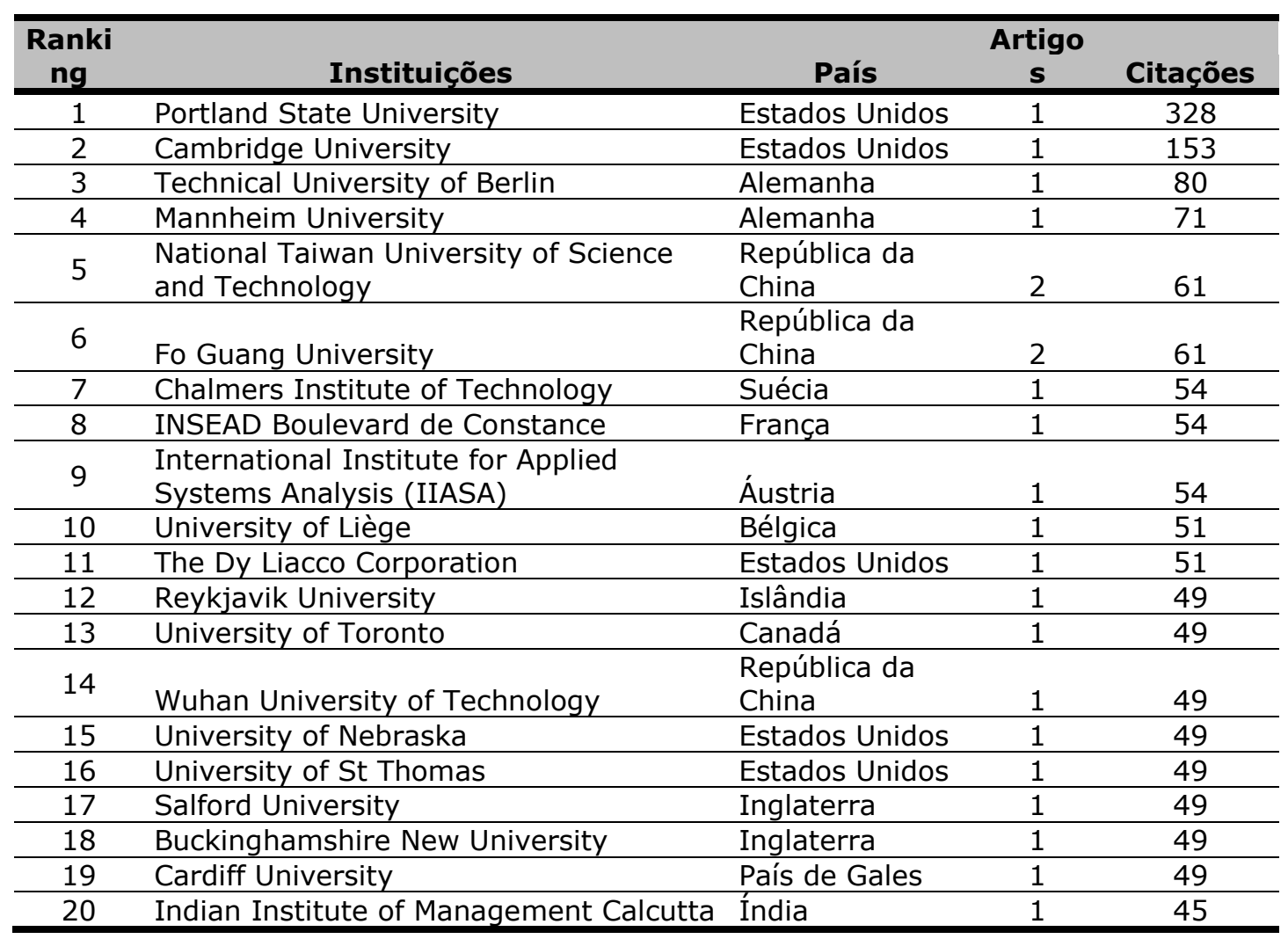

Fonte: elaborado pelos autores

\subsection{ANÁLISE SEMÂNTICA E DE CONTEÚDO}

\subsubsection{Palavras-chave}

Como o número de palavras-chave varia de um artigo a outro, optou-se por selecionar três palavras-chave de cada. Para essa seleção foi dada preferência ao assunto tratado, ou seja, dinâmica de sistemas e prospecção ou cenários, além das palavras relacionadas com a área ou assunto de aplicação do artigo analisado, quando presente.

$\mathrm{Na}$ Tabela 6, apresentam-se as variações das palavras-chave encontradas nos artigos no que tange à prospecção e dinâmica de sistemas. Foram encontradas nove variações com o termo previsão ou cenários, totalizando 22 referências a essas palavras-chave. Já dinâmica de sistemas apresentou sete variações, totalizando 29 referências. Observa-se também que os termos mais usados como palavras-chave foram previsão (6) e 
dinâmica de sistemas (20), para a primeira e a segunda palavra-chave, respectivamente.

Tabela 6: Classificação das palavras-chave

\begin{tabular}{lccc}
\hline Palavra-chave & Variações & Subtotal & Total \\
\hline & Prospecção tecnológica & 1 & 1 \\
& Previsão de telecomunicações & 1 & \\
Previsão do número de físicos & Análise de cenários & 1 & 22 \\
& Cenários & 1 & \\
& Previsão & 3 & \\
& Método de previsão & 1 & \\
& Avaliação de previsão & 4 & \\
& Previsão de demanda & 1 & \\
& Tendência & 1 & \\
& Modelo de previsão & 1 & \\
& Curva de crescimento & 20 \\
Dinâmica de sistemas & Dinâmica de sistemas & 4 \\
& Simulação & 1 \\
& Dinâmica de sistemas e simulação & 1 & 29 \\
& Simulação computacional & 1 \\
& Modelagem matemática & 1 \\
\end{tabular}

Fonte: elaborado pelos autores

$\mathrm{Na}$ Tabela 7, apresenta-se a relação das áreas e dos assuntos abordados pelos artigos, a partir da análise de palavras-chave, que permitiu classificá-las por áreas e assuntos de aplicação. Foram identificados 11 assuntos ou áreas: meio ambiente, logística, indústria, mercado/econômico, matemática, tecnologia, recursos humanos, riscos, gerenciamento, inovação e governança. Uma palavra-chave pode fazer parte de mais de um assunto ou área. Assim, algumas palavras-chave identificadas como relacionadas a previsão/cenários ou dinâmica de sistemas (Tabela 6) também se encaixam na classificação da Tabela 7, remeterem às áreas ou aos assuntos identificados. A fim de evitar a má interpretação de uma palavra-chave e, consequentemente, sua classificação errônea, na Tabela 7, o conteúdo dos artigos foi considerado no momento da classificação.

Nota-se a predominância de palavras-chave relacionadas a mercado e economia, com 11 variações encontradas, com destaque para previsão de demanda (quatro ocorrências). Essas palavras-chave foram usadas 14 vezes ao total. Em seguida, destacam-se assuntos relacionados à logística, com cinco variações encontradas, totalizando nove ocorrências, com destaque para cadeia de suprimentos (cinco ocorrências). Outra área de 
destaque é em gerenciamento, com oito palavras-chave diferentes, cada uma aparecendo uma vez.

Outros assuntos de destaque foram na área matemática, com o total de seis palavras-chave variadas, cada uma com uma ocorrência. Algumas aplicações ocorreram na indústria, com destaque para a indústria automobilística, com quatro ocorrências. A outra palavra-chave encontrada foi na indústria aérea.

Outras áreas e assuntos encontrados foram em tecnologia, meio ambiente, recursos humanos, riscos, inovação e governança. Essas classificações apresentaram quatro, quatro, três, duas, uma e uma variações de palavras-chave, respectivamente. 
Tabela 7: Classificação das palavras-chave por área e assunto

\begin{tabular}{|c|c|c|c|}
\hline Área/Assunto & Variações & $\begin{array}{l}\text { Subtot } \\
\text { al }\end{array}$ & Total \\
\hline \multirow{4}{*}{$\begin{array}{c}\text { Meio } \\
\text { Ambiente }\end{array}$} & Desperdício municipal de resíduos sólidos & 1 & \multirow{4}{*}{4} \\
\hline & Mudança climática & 1 & \\
\hline & Pegada ecológica & 1 & \\
\hline & Catástrofes & 1 & \\
\hline \multirow{7}{*}{ Logística } & & 5 & \multirow{7}{*}{9} \\
\hline & Cadeia de suprimentos & 1 & \\
\hline & Gerenciamento de inventário & 1 & \\
\hline & Transporte & & \\
\hline & Previsão de carga & & \\
\hline & Otimização & 1 & \\
\hline & & 1 & \\
\hline \multirow{5}{*}{ Indústria } & Indústria automotiva & 1 & \multirow{5}{*}{5} \\
\hline & Transporte & 1 & \\
\hline & Veículos elétricos e a combustível & 1 & \\
\hline & Indústria automobilística & 1 & \\
\hline & Indústria aérea & 1 & \\
\hline \multirow{11}{*}{$\begin{array}{l}\text { Mercado/Econô } \\
\text { mico }\end{array}$} & Estrutura de mercado & 1 & \multirow{11}{*}{14} \\
\hline & Competição de mercado & 1 & \\
\hline & Mercado de eletricidade & 1 & \\
\hline & Performance industrial & 1 & \\
\hline & Ciclo de negócios & 1 & \\
\hline & Macroeconomia & 1 & \\
\hline & Previsão de demanda & 4 & \\
\hline & Confiabilidade da demanda & 1 & \\
\hline & Demanda de energia & 1 & \\
\hline & Econometria & 1 & \\
\hline & Previsão de telecomunicações & 1 & \\
\hline \multirow{7}{*}{ Matemática } & Modelagem matemática & 1 & \multirow{7}{*}{7} \\
\hline & Filtro Kalman & 1 & \\
\hline & Modelo de Lotka-Volterra & 1 & \\
\hline & Filtro estendido de Kalman & 1 & \\
\hline & Simulação computacional & 1 & \\
\hline & Estimativa hierárquica & 1 & \\
\hline & Programação matemática & 1 & \\
\hline \multirow{4}{*}{ Tecnologia } & Tecnologia & 1 & \multirow{4}{*}{4} \\
\hline & Análise tecnológica orientada para o futuro & 1 & \\
\hline & Desenvolvimento tecnológico & 1 & \\
\hline & Technology roadmap & 1 & \\
\hline \multirow{3}{*}{$\begin{array}{l}\text { Recursos } \\
\text { Humanos }\end{array}$} & Planejamento de recursos humanos & 1 & \multirow{3}{*}{3} \\
\hline & Previsão do número de médicos & 1 & \\
\hline & Falta de médicos & 1 & \\
\hline \multirow{2}{*}{ Riscos } & Análise de riscos e probabilidade & 1 & \multirow{2}{*}{2} \\
\hline & Tomada de decisão baseada em riscos & 1 & \\
\hline \multirow{8}{*}{ Gerenciamento } & Tomada de decisão & 1 & \multirow{8}{*}{8} \\
\hline & Tomada de decisão baseada em riscos & 1 & \\
\hline & Decisão & 1 & \\
\hline & Satisfação do consumidor & 1 & \\
\hline & Planejamento de infraestrutura & 1 & \\
\hline & Investimento de capacidade & 1 & \\
\hline & Incertezas & 1 & \\
\hline & Resolução de problemas por análise de feedback & 1 & \\
\hline Inovação & Sistemas de inovação & 1 & 1 \\
\hline Governança & Governança da ciência & 1 & 1 \\
\hline
\end{tabular}

Fonte: elaborado pelos autores 


\subsubsection{Metodologia utilizada}

Na Tabela 8, resumem-se os principais métodos utilizados. Percebese que alguns artigos, também considerados na análise, utilizam mais de uma metodologia para sua elaboração. Observa-se que o método mais utilizado foi o de dinâmica de sistemas, incluindo modelagem e simulação, com o total de 27 aplicações. Em segundo lugar, seis artigos utilizaram a metodologia de estudo de caso. Em seguida, a metodologia mais utilizada foi a aplicação do Filtro de Kalman, visto como um método de linearização estatística para dinâmicas fortemente não lineares (Evensen, 1992; Miller, Ghil \& Gauthiez, 1994; Bouttier, 1994, citado por Burges, Leeuwen \& Evensen, 1998). Outras metodologias apresentadas foram: revisão de literatura, programação matemática, econometria, análise de tendências, outros métodos de modelagem, modelo de Lotka-Volterra, interpretação de estudos de previsão, technology roadmap e pesquisa exploratória.

\section{Tabela 8: Metodologias utilizadas nos artigos}

\begin{tabular}{ll}
\hline \multicolumn{1}{c}{ Metodologia } & Total \\
\hline Dinâmica de sistemas & 27 \\
\hline Estudo de caso & 6 \\
\hline Filtro de Kalman & 2 \\
\hline Revisão da literatura & 1 \\
\hline Pegada ecológica & 1 \\
\hline Programação matemática & 1 \\
\hline Econometria & 1 \\
\hline Análise de tendências & 1 \\
\hline Métodos de modelagem & 1 \\
\hline Modelo de Lotka-Volterra & 1 \\
\hline Interpretação de estudos de & 1 \\
\hline previsão & 1 \\
\hline Technology roadmap & 1 \\
\hline Pesquisa exploratória &
\end{tabular}

Fonte: elaborado pelos autores

\subsubsection{Pesquisas futuras em dinâmica de sistemas e prospecção de cenários}

Entre os 35 artigos pesquisados, foi possível identificar as oportunidades de pesquisa apontadas por alguns autores no que tange à dinâmica de sistemas, à prospecção de cenários e ao conjunto de ambos. $\mathrm{Na}$ Tabela 9, relacionam-se os autores que mencionaram estudos futuros 
nesses temas. Em relação ao uso conjunto de dinâmica de sistemas e prospecção, os autores propõem estudos relacionados ao uso de dinâmica de sistemas para a prospecção do futuro, com a abrangência dos modelos de simulação. Randers e Göluke (2007), como já mencionado, questionam se dinâmica de sistemas pode ser usada para fazer previsões e, caso positivo, se deveria ser usada.

Tabela 9: Oportunidades de pesquisa apontadas nos artigos

\begin{tabular}{|c|c|c|c|}
\hline & Autor & Ano & Oportunidade de pesquisa \\
\hline \multirow{5}{*}{ 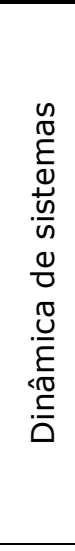 } & $\begin{array}{l}\text { Liehr, Grössler, Klein } \\
\text { e Milling }\end{array}$ & 2001 & $\begin{array}{l}\text { Expansão do modelo adotado para englobar a } \\
\text { área financeira }\end{array}$ \\
\hline & Randers e Göluke & 2007 & $\begin{array}{l}\text { Melhores práticas em dinâmica de sistemas } \\
\text { É essencial ter uma hipótese dinâmica para } \\
\text { quiar o trabalho de modelagem? }\end{array}$ \\
\hline & Daim et al. & 2006 & $\begin{array}{l}\text { Aplicação da metodologia Delphi com } \\
\text { especialistas para aumentar a capacidade do } \\
\text { modelo dinâmico em medir a difusão de } \\
\text { tecnologia }\end{array}$ \\
\hline & Warr e Ayres & 2006 & Melhoria dos loops de feedback dinâmicos \\
\hline & $\begin{array}{l}\text { Campuzano, Mula e } \\
\text { Peidro }\end{array}$ & 2010 & $\begin{array}{l}\text { Estudo de novas versões do modelo, } \\
\text { considerando n estágios e } \mathrm{n} \text { itens da cadeia de } \\
\text { suprimentos }\end{array}$ \\
\hline 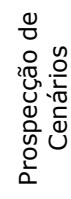 & Geum et al. & 2014 & $\begin{array}{l}\text { Incorporação da previsão de cenários no } \\
\text { technology roadmap }\end{array}$ \\
\hline \multirow{5}{*}{ 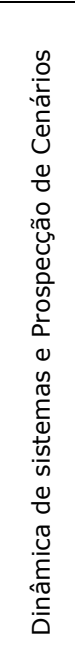 } & Randers e Göluke & 2007 & $\begin{array}{l}\text { Dinâmica de sistemas pode ser usada para } \\
\text { previsão e, caso positivo, deveria ser? }\end{array}$ \\
\hline & Liehr et al. & 2001 & $\begin{array}{l}\text { Implementação dos resultados da previsão } \\
\text { geradas pelo modelo dinâmico }\end{array}$ \\
\hline & $\begin{array}{l}\mathrm{Li}, \mathrm{Xu} \text {, Chen e } \\
\text { Simonovic }\end{array}$ & 2010 & $\begin{array}{l}\text { Extensão do modelo para considerar a variação } \\
\text { espacial do clima, a fim de aumentar a } \\
\text { habilidade de previsão do modelo }\end{array}$ \\
\hline & Geum et al. & 2014 & $\begin{array}{l}\text { Desenvolvimento de guias sobre como construir } \\
\text { um diagrama de loop causal na formulação de } \\
\text { cenários } \\
\text { Como diagramas de loops causais diferem de } \\
\text { acordo com o tipo de cenário } \\
\text { Ampliação do modelo proposto para mais } \\
\text { cenários no ramo de carros partilhados }\end{array}$ \\
\hline & $\begin{array}{l}\text { Neumann, Santa- } \\
\text { Eulalia, Yoshino e } \\
\text { Klasen }\end{array}$ & 2014 & $\begin{array}{l}\text { Investigação de pressupostos que influenciam o } \\
\text { comportamento de uma previsão, a partir de um } \\
\text { modelo baseado em dinâmica de sistemas }\end{array}$ \\
\hline
\end{tabular}

Fonte: elaborado pelos autores 


\section{CONCLUSÃO}

Diante do exposto, a área de prospecção de cenários vem se desenvolvendo ao longo dos últimos anos, principalmente nas duas últimas décadas com a chegada da linguagem computacional. Quando analisada a distribuição cronológica da presente pesquisa, para a qual foram selecionados apenas os artigos mais citados, é notório o crescimento de publicações nos últimos anos, principalmente em torno de temas que envolvem ferramentas de previsão do futuro como partida para tomadas de decisão. Tal fenômeno justifica-se pelo fato de que hoje não há mais margens para desperdícios nos setores públicos, privado e acadêmico na comparação com décadas passadas. Assim, instituições que têm maduro no seu dia a dia o tema do presente estudo, apresentam certo diferencial frente a qualquer tipo de competidor, uma vez que tomam decisões preventivas, estratégicas e com alto grau de assertividade.

Com relação aos autores, apesar da importância do tema, notou-se que não há um especialista na área de conhecimento de simulação e prospecção de cenários, quando se analisa o pilar de publicações de artigos com significativo número de citações. É fato que há um crescimento em publicações na área. No entanto, a cada nova publicação, é conhecido um novo autor, pois as publicações são realizadas por autores diferentes.

Conforme ilustrado na Tabela 3, os três autores que obtiveram mais de uma publicação na área foram responsáveis por apenas duas obras, as quais foram construídas em conjunto, também pelos três. Quando a análise dos autores é realizada pela ótica das citações vinculadas a cada autor, obtém-se outro resultado interessante. Considerando-se o número de citações dos dois artigos publicados por Suryani et al. (2010), 61 no total, esse número é menor que as citações obtidas pelos quatro primeiros artigos da Tabela 4, publicados por Daim et al. (2006), Lyneis (2000), Karavezyris et al. (2002) e Liehr et al. (2001), com respectivas 328, 153, 80 e 71 citações. Nessa outra ótica, confirma-se o resultado de que não há um especialista no assunto, quando se analisa sob a perspectiva de artigos de grande impacto publicados na área de simulação e prospecção e cenários. 
Ainda com relação aos autores, outro fato curioso diz respeito ao trabalho em conjunto nas publicações dos artigos. Dos 35 artigos analisados, apenas sete foram elaborados por apenas um autor. Ou seja, $80 \%$ dos artigos foram escritos em conjunto por dois ou mais autores. Esses dados são, de certa forma, positivos, uma vez que ideias discutidas em conjunto proporcionam discussões e alinhamentos para que o resultado final, ou seja, o artigo, tenha perfeita sintonia com o propósito para o qual ele foi concebido.

Outro fator relevante, sob a ótica do trabalho em conjunto para elaboração dos artigos na área de estudo, diz respeito às diferentes nacionalidades dos autores em um mesmo artigo. Para ilustrar esse fato, tem-se como exemplo o artigo "Modeling technological innovation risks of an entrepreneurial team using system dynamics: An agent-based perspective", que foi elaborado por cinco autores de três diferentes nacionalidades, inclusive em diferentes continentes, entre eles o islandês Wu, os chineses Kefan, Hua e Shi e o americano Olson (2010). Outro exemplo dessa disseminação pode ser observado na obra "Streamflow forecast and reservoir operation performance assessment under climate change" (Li et al., 2010), elaborada por chineses e canadenses, e na obra "Supporting cognitive feedback using system dynamics: A demand model of the global system of mobile telecommunication" (Bui \& Loebbecke, 1996), produzida por autores da Alemanha e de Hong Kong.

A distribuição de autores publicados por países demonstra uma concentração nos Estados Unidos, Alemanha e Reino Unido. Os dois primeiros países são também aqueles cujas instituições de pesquisa possuem maior número de citações, o que pode evidenciar um desenvolvimento maior nessa área do conhecimento nessas localidades.

Notou-se que os trabalhos mais citados estão dispersos em 25 periódicos, o que evidencia que não há uma concentração expressiva. Os periódicos mais recorrentes são, entretanto, aqueles mais alinhados com o objeto de pesquisa, cujas pesquisas tratam, sobretudo, dos métodos e práticas para a simulação e prospecção de cenários. A dispersão pode ocorrer, entre outros motivos, pela aplicação do tema aqui estudado em 
áreas específicas do conhecimento, as quais são publicadas em periódicos relevantes para o assunto em questão.

Em relação às palavras-chave, observa-se que muitos artigos apresentaram termos relacionados à prospecção de cenários, dinâmica de sistemas e suas variações. Alguns artigos, como os de Bui e Loebbecke (2010); Campuzano, Mula e Peidro (2010); Gordon e Stover (1976); Kumar e Yamaoka (2007); Suryani, Chou, Hartono e Chen (2010) e Winch (1999) repetem palavras-chave que se encaixam na mesma variação apresentada na Tabela 6.

Muitas palavras-chave foram identificadas na área de mercado e economia. Isso ocorre porque alguns artigos utilizaram dinâmica de sistemas para elaborar previsões sobre mercados e economia. Liehr et al. (2001) utilizam dinâmica de sistemas para analisar e gerenciar o ciclo de negócios de linhas aéreas. Ishikawa, Ohba, Yokooka, Nakamura e Ogasawara (2013) propõem um modelo de simulação da capacidade de investimento no mercado de eletricidade, baseando-se na previsão do preço desta. Sterman (1988) elaborou um modelo para explicar a história da previsão de demanda de energia nos Estados Unidos. Ashayeri e Lemmes (2006) desenvolvem um modelo para prever a demanda da cadeia de suprimentos. Na mesma linha de aplicação de previsão na cadeia de suprimentos, tem-se Kamath e Roy (2007); Campuzano et al. (2010); Barlas e Gunduz (2011).

Na área econômica, no que diz respeito à previsão de demanda, podem-se citar Bui e Loebbecke (2010), que pesquisam da previsão de demanda de passageiros em terminais aéreos; Suryani, Chou, Hartono e Chen (2010), propõem modelos de previsão de demanda relacionados a políticas de expansão; Barre (2014), que elabora um modelo, utilizando recursos de dinâmica de sistemas, para prever inovações radicais.

Em relação ao meio ambiente, destacam-se quatro artigos. Judd (2003) aplica a dinâmica de sistemas na definição de pegada ecológica, que corresponde a uma medida do que a humanidade requer da natureza (Footprint, 2014), a fim de propor medidas sustentáveis. Buongiorno (1996) utiliza dinâmica de sistemas para prever cenários climáticos. Syntetos, Boylan e Disney (2009) aplicam dinâmica de sistemas na indústria 
automotiva para explorar cenários relacionados a redução, reutilização e reciclagem. Observa-se pelas datas dos artigos que a aplicação de dinâmica de sistemas e a prospecção de cenários relacionados ao meio ambiente são mais recentes, com a primeira ocorrência em 2007. Uma proposta de estudo futura é promover a análise de artigos que relacionam o tema meio ambiente com dinâmica de sistemas e prospecção de cenários. Como o artigo de Syntetos et al. (2009), pode-se desenvolver também projetos em outras indústrias a fim de estabelecer essa relação de redução, reutilização e reciclagem.

Muito embora tenha como objetivo de estudo elaborar um panorama das publicações, o presente trabalho não o faz sem algumas limitações. Em primeiro lugar, apenas artigos de periódicos acadêmicos indexados nas bases de dados Scopus e Scielo foram incluídos na análise, uma vez que se acredita que ambas forneçam uma visão abrangente do tema pesquisado. $A$ própria escolha da análise com base apenas em artigos científicos é outra limitação da presente pesquisa: embora estes sejam, geralmente, considerados o mais alto nível de pesquisa para aquisição de informações e disseminação de novas descobertas (Vieira, 2012), outros conhecimentos sobre o tema podem ser encontrados em anais de eventos, dissertações de mestrado, teses de doutorado, livros, etc. Considerando-se essas fontes de conhecimento, os resultados e as conclusões podem ser alterados.

Em segundo lugar, alguns artigos publicados e escolhidos como parte importante da coleção de publicações não estavam disponíveis para leitura ou simplesmente o texto completo não foi encontrado nos periódicos indexados. Uma terceira potencial limitação está relacionada com a escolha dos termos para a pesquisa bibliográfica. Embora se acredite que os construtos utilizados sejam adequados, é possível que outros trabalhos não tenham sido selecionados em virtude dos termos utilizados no título ou resumo. E, finalmente, apesar de um esforço consciente para identificar o tema principal e a abordagem de cada artigo, a análise e classificação das publicações foi realizada de forma subjetiva, baseada no conhecimento dos autores sobre o assunto. 


\section{REFERÊNCIAS}

Araújo, C. A. (2006). Bibliometria: evolução histórica e questões atuais. Em Questão, 12(1).

Ashayeri, J., \& Lemmes, L. (2006). Economic value added of supply chain demand planning: a system dynamics simulation. Robotics and Computer-Integrated Manufacturing, 22(5), 550-556.

Barlas, Y., \& Gunduz, B. (2011). Demand forecasting and sharing strategies to reduce fluctuations and the bullwhip effect in supply chains. Journal of the Operational Research Society, 62(3), 458-473.

Barre, R. (2014). Innovation systems dynamics and the positioning of Europe. A review and critique of recent Foresight studies. Foresight, 16(2), 126-141.

Bishop, P., Hines, A., \& Collins, T. (2007). The current state of scenario development: an overview of techniques. Foresight, 9(1), 5-25.

Blackman, A. W. (1971). Forecasting through dynamic modeling. Technological Forecasting and Social Change, 3, 291-307.

Börjeson, L., Höjer, M., Dreborg, K. H., Ekvall, T., \& Finnveden, G. (2006). Scenario types and techniques: towards a user's guide. Futures, 38(7), 723-739.

Bradfield, R., Wright, G., Burt, G., Cairns, G., \& Van Der Heijden, K. (2005). The origins and evolution of scenario techniques in long range business planning. Futures, 37(8), 795-812.

Bueno, N. P. (2013). Identificando mudanças de regimes sistêmicos em processos econômicos: um procedimento baseado na abordagem de dinâmica de sistemas. Economia e Sociedade, 22(1), 77-106.

Bui, T., \& Loebbecke, C. (1996). Supporting cognitive feedback using system dynamics: a demand model of the global system of mobile telecommunication. Decision Support Systems, 17(2), 83-98.

Buongiorno, J. (1996). Forest sector modeling: a synthesis of econometrics, mathematical programming, and system dynamics methods. International Journal of Forecasting, 12(3), 329-343.

Burgers, G., Jan van Leeuwen, P., \& Evensen, G. (1998). Analysis scheme in the ensemble Kalman filter. Monthly Weather Review, 126(6), 17191724.

Campuzano, F., Mula, J., \& Peidro, D. (2010). Fuzzy estimations and system dynamics for improving supply chains. Fuzzy Sets and Systems, 161(11), 1530-1542. 
Costa, L., Marinho, S. V. (2017). A utilização de ferramentas prospectivas em gerenciamento de risco de projetos estratégicos-análise dos institutos de inovação e tecnologia do Sistema Fiesc. Future Studies Research Journal: Trends and Strategies, 9(2), 88-115.

Cuhls, K. (2003). From forecasting to foresight processes - new participative foresight activities in Germany. Journal of Forecasting, 22(2-3), 93-111.

Daim, T. U., Rueda, G., Martin, H., \& Gerdsri, P. (2006). Forecasting emerging technologies: Use of bibliometrics and patent analysis. Technological Forecasting and Social Change, 73(8), 981-1012.

Elsevier. (2014). Scopus. Recuperado em 7 de outubro, 2014, de http://www.elsevier.com/online-tools/scopus/

Elsevier. (2017). Scopus: Content Coverage Guide. Retrieved on February 6 2018, from https://www.elsevier.com/_data/assets/pdf_file/0007/69451/0597Scopus-Content-Coverage-Guide-US-LETTER-v4-HI-singles-no-ticks.pdf

Ensslin, L., Ensslin, S. R., Lacerda, R. T. O., \& Tasca, J. E. ProKnow-C, knowledge development process-constructivist: processo técnico com patente de registro pendente junto ao INPI. Brasil, 2010.

Evensen, G. (1992). Using the extended Kalman filter with a multilayer quasi-geostrophic ocean model. Journal of Geophysical Research: Oceans, 97(C11), 17905-17924.

Featherston, C. R.; Doolan, M. (2013). Using system dynamics to inform scenario planning: a case study. Proceedings of International Conference of the System Dynamics Society, 30, St. Gallen, Switzerland.

Figueiredo, J. C. B. D. (2009). Estudo da difusão da tecnologia móvel celular no Brasil: uma abordagem com 0 uso de Dinâmica de Sistemas. Produção, 19(1), 230-245.

Footprint (2014). Footprint basics: overview. Recuperado em 5 de outubro, 2014, de http://www.footprintnetwork.org/en/index.php/GFN/page/footprint_basic s_overview/

Forrester, J. W. (1961). Industrial dynamics. Cambridge: MIT Press.

Geum, Y., Lee, S., \& Park, Y. (2014). Combining technology roadmap and system dynamics simulation to support scenario-planning: a case of carsharing service. Computers \& Industrial Engineering, 71, 37-49.

Godet, M. (2000). The art of scenarios and strategic planning: tools and pitfalls. Technological Forecasting and Social Change, 65(1), 3-22. 
Gordon, T. J., \& Stover, J. (1976). Using perceptions and data about the future to improve the simulation of complex systems. Technological Forecasting and Social Change, 9(1-2), 191-211.

Hood, W., \& Wilson, C. (2001). The literature of bibliometrics, scientometrics, and informetrics. Scientometrics, 52(2), 291-314.

Huss, W. R., \& Honton, E. J. (1987). Scenario planning-What style should you use?. Long Range Planning, 20(4), 21-29.

Ishikawa, T., Ohba, H., Yokooka, Y., Nakamura, K., \& Ogasawara, K. (2013). Forecasting the absolute and relative shortage of physicians in Japan using a system dynamics model approach. Human Resources for Health, 11(1), 41.

Judd, K. (2003). Nonlinear state estimation, indistinguishable states, and the extended Kalman filter. Physica D: Nonlinear Phenomena, 183(3-4), $273-281$.

Kamath, N. B., \& Roy, R. (2007). Capacity augmentation of a supply chain for a short lifecycle product: a system dynamics framework. European Journal of Operational Research, 179(2), 334-351.

Karavezyris, V., Timpe, K. P., \& Marzi, R. (2002). Application of system dynamics and fuzzy logic to forecasting of municipal solid waste. Mathematics and Computers in Simulation, 60(3), 149-158.

Krippendorff, K. (2013). Content analysis: an introduction to its methodology. Thousand Oaks: Sage.

Kumar, S., \& Yamaoka, T. (2007). System dynamics study of the Japanese automotive industry closed loop supply chain. Journal of Manufacturing Technology Management, 18(2), 115-138.

Kwakkel, J. H., \& Pruyt, E. (2013). Exploratory Modeling and Analysis, an approach for model-based foresight under deep uncertainty. Technological Forecasting and Social Change, 80(3), 419431.

Lakka, S., Michalakelis, C., Varoutas, D., \& Martakos, D. (2013). Competitive dynamics in the operating systems market: modeling and policy implications. Technological Forecasting and Social Change, 80(1), 88-105.

Li, L., Xu, H., Chen, X., \& Simonovic, S. P. (2010). Streamflow forecast and reservoir operation performance assessment under climate change. Water Resources Management, 24(1), 83-104.

Liehr, M., Grössler, A., Klein, M., \& Milling, P. M. (2001). Cycles in the sky: understanding and managing business cycles in the airline market. System Dynamics Review, 17(4), 311. 
Lyneis, J. M. (2000). System dynamics for market forecasting and structural analysis. System Dynamics Review, 16(1), 3.

Martin, B. R. (2010). The origins of the concept of 'foresight' in science and technology: an insider's perspective. Technological Forecasting and Social Change, 77(9), 1438-1447.

Martin, B. R., \& Johnston, R. (1999). Technology foresight for wiring up the national innovation system: experiences in Britain, Australia, and New Zealand. Technological Forecasting and Social Change, 60(1), 37-54.

Martino, J. P. (1993). Technological forecasting for decision making. Ohio: McGraw-Hill.

Miller, R. N., Ghil, M., \& Gauthiez, F. (1994). Advanced data assimilation in strongly nonlinear dynamical systems. Journal of the Atmospheric Sciences, 51(8), 1037-1056.

Neumann, D., Santa-Eulalia, L. A. D., Yoshino, R. T., \& Klasen, J. (2014). A novel demand forecasting model for radical innovation. Production, 24(3), 605-617.

Pidd, M. (1998). Computer simulation in management science. Lancaster: John Wileys \& Sons.

Randers, J., \& Goluke, U. (2007). Forecasting turning points in shipping freight rates: lessons from 30 years of practical effort. System Dynamics Review, 23(2), 253-284.

Rousseaux, P., Van Cutsem, T., \& Dy Liacco, T. E. (1990). Whither dynamic state estimation? International Journal of Electrical Power \& Energy Systems, 12(2), 104-116.

Saritas, O., \& Aylen, J. (2010). Using scenarios for roadmapping: the case of clean production. Technological Forecasting and Social Change, 77(7), 1061-1075.

Schoemaker, P. J. (1995). Scenario planning: a tool for strategic thinking. Sloan Management Review, 36(2), 25.

Schnaars, S. P. (1987). How to develop and use scenarios. Long Range Planning, 20(1), 105-114.

Sterman, J. D. (1988). Modeling the formation of expectations: the history of energy demand forecasts. International Journal of Forecasting, 4(2), 243-259.

Sterman, J. D. (2000). Business dynamics: systems thinking and modeling for a complex world. New York: McGraw-Hill.

Sterman, J. D. (2007). Exploring the next great frontier: system dynamics at fifty. System Dynamics Review, 23(2-3), 89-93. 
Suryani, E., Chou, S. Y., Hartono, R., \& Chen, C. H. (2010). Demand scenario analysis and planned capacity expansion: a system dynamics framework. Simulation modelling practice and theory, 18(6), 732-751.

Suryani, E., Chou, S. Y., \& Chen, C. H. (2010). Air passenger demand forecasting and passenger terminal capacity expansion: a system dynamics framework. Expert Systems with Applications, 37(3), 23242339.

Syntetos, A. A., Boylan, J. E., \& Disney, S. M. (2009). Forecasting for inventory planning: a 50-year review. Journal of the Operational Research Society, 60(1), S149-S160.

Vieira, C. L. S. (2012). Proposta de um modelo de implantação de tecnologias de informação e comunicação para prestadores de serviços logísticos. Dissertação de Mestrado, Universidade Federal de Santa Catarina: Florianópolis, SC, Brasil.

Warr, B., \& Ayres, R. (2006). REXS: A forecasting model for assessing the impact of natural resource consumption and technological change on economic growth. Structural Change and Economic Dynamics, 17(3), 329-378.

Weber, R. P. (1990). Basic content analysis. London: Sage.

Winch, G. (1999). Dynamic visioning for dynamic environments. Journal of the Operational Research Society, 50(4), 354-361.

Wu, D. D., Kefan, X., Hua, L., Shi, Z., \& Olson, D. L. (2010). Modeling technological innovation risks of an entrepreneurial team using system dynamics: an agent-based perspective. Technological Forecasting and Social Change, 77(6), 857-869.

Zentner, R. (1982). Scenarios, past, present and future. Long Range Planning, 15(3), 12-20. 